\title{
MINIMAL SPLITTING FIELDS IN CYCLOTOMIC EXTENSIONS
}

\author{
EUGENE SPIEGEL AND ALLAN TROJAN
}

\begin{abstract}
Suppose $G$ is a finite group of exponent $n$ and $X$ an irreducible character of $G$. In this note we give sufficient conditions for the existence of a minimal degree splitting field $L$ with $Q(X) \subseteq L \subseteq Q\left(\zeta_{n}\right)$.
\end{abstract}

1. Introduction. Let $G$ be a finite group of exponent $n, X$ an irreducible complex character of $G, m_{Q}(X)$ the Schur index of $X$ and $A(X ; Q)$ the simple component of the group algebra $Q G$ corresponding to $X$. In this note we investigate the existence of a splitting field $L$ of $X$ such that $Q(X) \subseteq L \subseteq Q\left(\zeta_{n}\right)$ and $[L: Q(X)]=m_{Q}(X)$, where $\zeta_{n}$ denotes a primitive $n$th root of unity. Fein $[3,4]$ and more recently Mollin [9] have investigated this question showing that such $L$ does not always exist, and giving sufficient conditions when this minimal splitting field does exist. An improved sufficient condition for the existence of $L$ is given, as an application of Abhyankar's Lemma. The authors would like to thank Dr. Gary Cornell for both pointing out Abhyankar's Lemma and useful discussions about its applicability.

If $K$ is the algebraic number field $Q(X), q$ a rational prime and $q_{1}$ and $q_{2}$ primes in $K$ lying above $q$, then $A(X ; Q) \otimes_{K} K_{q_{1}}$ and $A(X, Q) \otimes_{K} K_{q_{2}}$ have the same index which we denote as ind ${ }_{q} A(X ; Q) . K_{q}$ denotes the $q_{1}$-adic completion of $K$.

For $n$ an integer and $p$ a prime we use $n_{p}$ to denote the $p$-part of $n$.

2. For completeness, we begin with Abhyankar's Lemma.

THEOREM. Let $F$ be a local field and $E_{1}$ and $E_{2}$ finite extensions of $F$ with ramification indices $e_{1}$ and $e_{2}$ respectively. Suppose $E_{2}$ is tamely ramified and $e_{2} \mid e_{1}$. Then $E_{1} E_{2}$ is an unramified extension of $E_{1}$.

Proof. We follow Cornell [2, Theorem 1, p. 83]. Let $L$ be the maximal unramified extension of $F$ in $E_{2}$, so that $E_{2}$ is totally unramified over $L$. Then $E_{1} L$ is unramified over $E_{1}$, and $e\left(E_{1} L \mid F\right)=e\left(E_{1} \mid F\right)$. Similarly $e\left(E_{1} L \mid L\right)=e\left(E_{1} \mid F\right)$. As the composite of an unramified extension with an unramified extension. remains unramified. we may assume that $E_{2} \mid F$ is a totally and tamely ramified extension. From [8, p. 249], there is a prime element $\pi \in F$ with $E_{2}=F\left(\pi^{1 / e_{2}}\right)$. Let $\Pi$ be a prime element in $E_{1}$, so $u \Pi^{e_{1}}=\pi$ for $u$ a unit in $E_{1}$. Then $E_{1} E_{2}=E_{1}\left(u^{1 / e^{2}}: \Pi^{e_{1} / e_{2}}\right)=E_{1}\left(u^{1} e_{2}\right)$ as $e_{2} \mid e_{1}$, and $E_{1} E_{2}$ is unramified over $E_{1}\left(u^{1 / e^{\prime}}\right)$ which is unramified over $E_{1}$. The result now follows.

Received by the editors December 4. 1981 and, in revised form. February 2. 1982. 1980 Mathematics Subject Classification. Primary 20C05. 
Let $K$ be a finite abelian extension of $Q$ and $q$ a rational prime. If $F=K \cap Q\left(\zeta_{q}\right)$ and $t=$ g.c.d. $\left(q-1, e_{q}(K \mid Q)\right)$, where $e_{q}(K \mid Q)$ denotes the index of ramification of $q$ in $K \mid Q$, then we denote by $E$ the unique subfield of $Q\left(\zeta_{q}\right)$ of degree $t$ over $Q$. $Q\left(\zeta_{q}\right)$ is a cyclic extension of $Q$ of degree $q-1 . E \supseteq F$ as $Q\left(\zeta_{q}\right)$ is totally ramified at $q$.

We then have the following diagram of fields

(*)

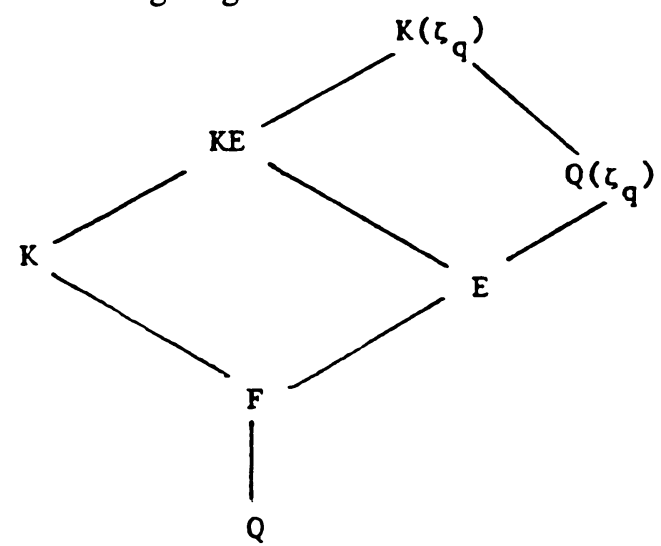

with $[K E: K]=[E: F]$ and $\left[K\left(\zeta_{q}\right): K E\right]=\left[Q\left(\zeta_{q}\right): E\right]$. By Abhyankar's Lemma, $K E$ is a totally unramified extension of $K$ with respect to $q$. If $L_{1}$ is an unramified extension at $q$ of $K E$ in $K\left(\zeta_{q}\right)$ and $L_{2}$ is the subfield of $Q\left(\zeta_{q}\right)$ containing $E$ with $\left[L_{2}: E\right]=\left[L_{1}: K E\right]$, then $K L_{2}=L_{1}$. Also $e_{q}(L \mid Q)=e_{q}(K \mid Q)$, and $e_{q}(K \mid Q)=$ $\left[L_{2}: Q\right] e_{q}\left(L_{1} \mid L_{2}\right)$. This implies $\left[L_{2}: Q\right] \mid e_{q}(K \mid Q)$ and as $t \mid\left[L_{2}: Q\right]$, we must have [ $\left.L_{2}: Q\right]=t$. Thus $L_{2}=E$ and $L_{1}=K E$ and $K\left(\zeta_{q}\right)$ is a totally ramified extension at $q$ of $K E$.

Let, now, $\bar{F}=K_{q} \cap Q_{q}\left(\zeta_{q}\right)$ and $\bar{t}=$ g.c.d. $\left(q-1\right.$, e $\left.\left(K_{q} \mid Q_{q}\right)\right)$, where $e\left(K_{q} \mid Q_{q}\right)$ denotes the ramification index of the local field $K_{q}$ over $Q_{q}$. Since $e\left(K_{q} \mid Q_{q}\right)=$ $e_{q}(K \mid Q), t=\bar{t}$. Let $\bar{E}$ denote the unique subfield of $Q_{q}\left(\zeta_{q}\right)$ of degree $t$ over $Q_{q}$. $\bar{E} \supseteq \bar{F}$ and $\left[Q_{q}\left(\zeta_{q}\right): E\right]=\left[Q\left(\zeta_{q}\right): E\right]$. This gives the following diagram of fields
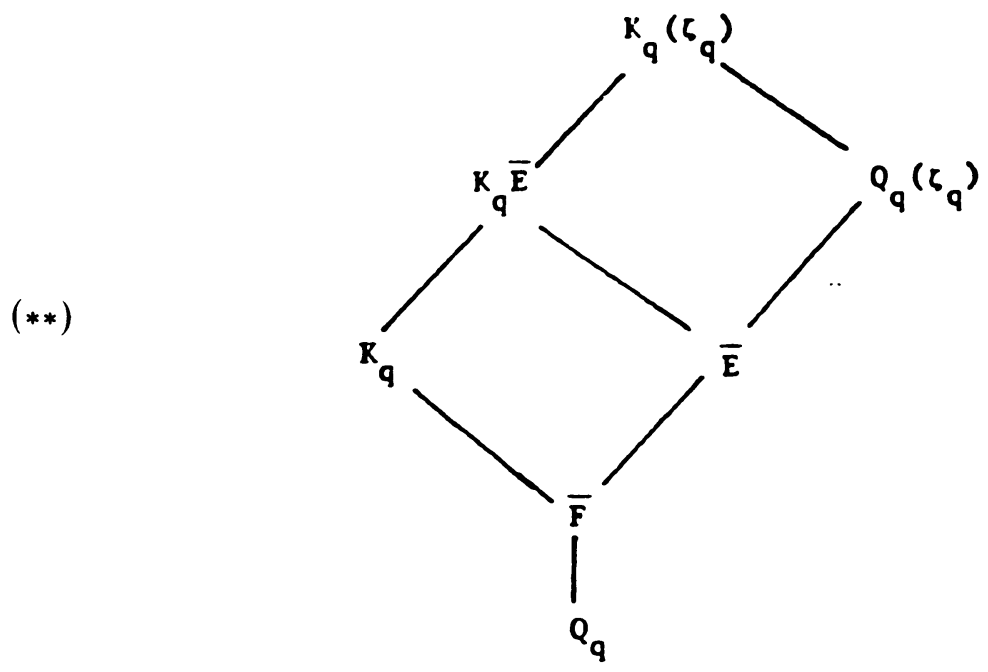
with $\left[K_{q} \bar{E}: K_{q}\right]=[\bar{E}: \bar{F}]$ and $\left[K_{q}\left(\zeta_{q}\right): K_{q} \bar{E}\right]=\left[Q\left(\zeta_{q}\right): E\right]$. Again by Abhyankar's Lemma, $K_{q} \bar{E}$ is an unramified extension of $K_{q}$ and $K_{q}\left(\zeta_{q}\right)$ is a totally ramified extension of $K_{q} \bar{E}$.

With the above notation, we have shown

Proposition 1. Let $p$ be a rational prime. The following are equivalent:

(a) $\left[K\left(\zeta_{q}\right): K\right]_{p}=\left[K_{q}\left(\zeta_{q}\right): K_{q}\right]_{p}$,

(b) $[E: F]_{p}=[\bar{E}: \bar{F}]_{p}$,

(c) $[F: Q]_{p}=\left[\bar{F}: Q_{q}\right]_{p}$.

CoRollary 2. If $p \nmid[E: F]$, then $e\left(K_{q}\left(\zeta_{q}\right) K_{q}\right)_{p}=\left[K_{q}\left(\zeta_{q}\right): K_{q}\right]_{p}=\left[K\left(\zeta_{q}\right) K\right]_{p}=$ $e_{q}\left(K\left(\zeta_{q}\right) K\right)_{p}$.

Proof. $p \nmid[E: F]$ implies $[E: F]_{p}=[\bar{E}: \bar{F}]_{p}=0$. The result then follows from the proposition, as $F \supseteq F_{q}$ and $K E / K$ and $K_{q} \bar{E} / K_{q}$ are totally unramified while $K\left(\zeta_{q}\right) / K E$ and $K_{q}\left(\zeta_{q}\right) / K_{q} \bar{E}$ are totally ramified at $q$.

THEOREM 3. Let $X$ be an irreducible character of a finite group $G$ of exponent $n$. Suppose $m_{Q}(X) \geqslant 3$ and $p$ is a prime with $p^{c} \| m_{Q}(X), c>0$. Let $r$ be the smallest positive integer such that $Q\left(\zeta_{r}\right) \supset Q(X)$ and $q_{1}, q_{2}, \ldots, q_{t}$ the distinct rational primes with [ind $\left.q_{1} A(X ; Q)\right]_{p}=p^{a_{i}}, a_{i} \geqslant 1$. Suppose that for each $q_{i}, i=1, \ldots, t$, we have $e_{q_{1}}\left(K\left(\zeta_{q_{1}}\right) / K\right)_{p}=\left[K\left(\zeta_{q_{1}}\right): K\right]_{p}$. Then there exists an extension field $L$ of $K$ contained in $Q\left(\zeta_{n}\right)$ with $[L: K]=p^{c}$ and $p \nmid \operatorname{ind}\left(L \otimes_{K} A(X, Q)\right)$.

Proof. Since $p^{c}=1$. c.m. $\left\{p^{a_{1}}, p^{a_{2}}, \ldots, p^{a_{i}}\right\}$ we have $a_{i} \leqslant c$ and $a_{i}=c$ for some $i$. We can suppose that $a_{1}=c$. By the theorem of Benard and Shacher [1], $\zeta_{p^{\prime}} \in K$.

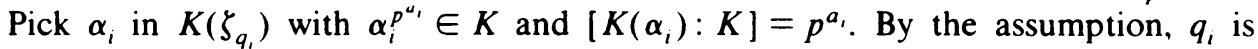
totally ramified in $K\left(\alpha_{i}\right) / K$ and $q_{j}$ is unramified in $K\left(\alpha_{i}\right) / K$ for $i \neq j$. Let $\alpha=\alpha_{1} \alpha_{2} \cdots \alpha_{t} . K\left(\alpha, \alpha / \alpha_{i}\right)=K\left(\alpha_{i}, \alpha / \alpha_{i}\right)$ implying $e_{q_{1}}(K(\alpha) / K)=p^{a_{i}}$ and thus $[K(\alpha): K]=p^{c}$. If $p^{c} \geqslant 3$ or all $q_{i}$ are odd, then $L=K(\alpha)$ is the desired splitting field since $A(X ; Q)$ has trivial index at any infinite prime. Similarly, if $p^{c}=2$ and all $q_{i}$ are odd. If $p=2$ and $q_{i}=2$ we find $L$ as in Mollin [9, p. 109].

In the following, $h(K)$ denotes the class number of $K$ and $Q\left(\zeta_{r}\right)$ denotes the smallest root of unity field containing $K$.

Corollary 4 (Mollin). Let $G$ be a finite group of exponent $n, X$ an irreducible character of $G$ and $m_{Q}(X) \geqslant 3$. Suppose that $p^{c} \| m(X)$ with $c>0$ and $p \nmid\left[Q\left(\zeta_{r}\right): K\right]$. Then there exists a field $L$ with $K \subset L \subset Q\left(\zeta_{n}\right),[L: K]=p^{c}$ and $p \nmid \operatorname{ind}\left(L \otimes_{K} A(X ; Q)\right)$.

Proof. Let $q_{1}, q_{2}, \ldots, q_{t}$ be the rational primes for which $p \mid$ ind $_{q_{1}} A(X ; Q)$. If $1 \leqslant i \leqslant t$, by the linear disjointness of full cyclotomic fields over $Q, Q\left(\zeta_{r}\right) \cap Q\left(\zeta_{q_{1}}\right)$ is either $Q\left(\zeta_{q_{1}}\right)$ or $Q$. If $Q\left(\zeta_{r}\right) \cap Q\left(\zeta_{q_{1}}\right)=Q\left(\zeta_{q_{1}}\right)$, then $A(X, Q) \otimes_{K} A\left(\zeta_{r}\right)=B$ is a central simple algebra with index still divisible by $p^{c}$, since $p \nmid\left[Q\left(\zeta_{r}\right): K\right]$. Similarly $p \mid$ ind $_{q_{1}} B$. But then $B \otimes K_{q_{i}}$, has a nontrivial index and contains a $q_{i}$ th root of unity in its center contradicting [10, Theorem 4.4]. Hence we must have $Q\left(\zeta_{r}\right) \cap Q\left(\zeta_{q_{1}}\right)=Q$ 
and $q_{i}$ is unramified in $K / Q$. Looking at $(*)$, we see that $E=F=Q$. From Corollary $2, e_{q_{1}}\left(K\left(\zeta_{q_{1}}\right) / K\right)_{p}=\left[K\left(\zeta_{q_{1}}\right): K\right]_{p}$ and the result follows via Theorem 3.

COROLl.ARY 5. Let $G$ be a finite group of exponent $n, X$ an irreducible character of $G$ and $m_{Q}(X) \geqslant 3$. Suppose that $p^{\prime} \| m(X), c>0$ and $p \nmid h(K)$. Then there exists a field $L$ with $K \subset L \subset Q\left(\zeta_{n}\right),[L: K]=p^{c}$ and $p \nmid \operatorname{ind}\left(L \otimes_{K} A(X: Q)\right)$.

Proof. From class field theory, $p \nmid h(K)$ implies there are no unramified abelian extensions of $K$ of degree $p$. By $(*), p \nmid[K E: K]$ and so $e_{q_{1}}\left(K\left(\zeta_{q_{1}}\right) / K\right)_{p}=$ $\left[K\left(\zeta_{q_{1}}\right): K\right]_{p}$ for any prime $q_{i}$. By Theorem 3 , the result follows.

These corollaries can be combined to give a generalization of Mollin's result [9. Corollary 1].

COROLlARY 6. Let $G$ be a finite group of exponent $n, X$ an irreducible character of $G$ and $m(X) \geqslant 3$. Suppose that $1=\left(m(X),\left[Q\left(\zeta_{r}\right): K\right], h(K)\right)$. Then there exists $a$ splitting field $L$ of $X$ with $[L: K]=m(X)$ and $K \subset L \subset Q\left(\zeta_{n}\right)$.

Proof. Write $m=p^{e_{1}} p_{2}^{e_{2}} \cdots p_{s}^{e^{2}}$. If $p_{i} \mid m(X)$ and $p \nmid h(K)$, find a field $L_{i}$ as in Corollary 5. If $p_{s} \mid(m(X), h(K))$, then $p_{j} \nmid\left[Q\left(\zeta_{r}\right): K\right]$ and find a field $L_{j}$ as in Corollary 4. Then $L=L_{1} L_{2} \cdots L_{s}$ is the desired splitting field.

The following extends Corollary 4.

COROLlaRY 7. Let $G$ be a finite group of exponent $n, X$ an irreducible character of $G . m(X) \geqslant 3$, and $p$ a prime with $p^{c} \| m(X), c>0$. Let $q_{1}, q_{2}, \ldots, q_{t}$ be the distinct rational primes such that $p \mid$ ind $_{q_{1}}(A(X ; Q))$ and suppose that $p \nmid e_{q_{1}}\left(Q\left(\zeta_{r}\right) \mid K\right)$. Then there exists a field $L$ with $K \subset L \subset Q\left(\zeta_{n}\right),[L: K]=p^{c}$ and $p \nmid \operatorname{ind}\left(L \otimes_{K} A(X ; Q)\right)$.

Proof. We claim that $Q\left(\zeta_{r}\right) \cap Q\left(\zeta_{q_{1}}\right)=Q$. Otherwise, we can assume that $q_{i}$ is odd and $Q\left(\zeta_{r}\right) \cap Q\left(\zeta_{q_{i}}\right)=Q\left(\zeta_{q_{1}}\right)$ and $q_{i} \mid r$. Write $n=q^{s} u$ with $(u, q)=1$. $\left(e\left(K_{q} \mid \zeta_{u}\right) K_{q}\right)=1$. As $q_{i}$ has ramification index relatively prime to $p$ in $Q\left(\zeta_{n}\right) \mid K$, also $\left(e\left(K_{q}\left(\zeta_{q^{\prime}}\right) \mid K_{q}\right), p\right)=1$. Let $I$ be the inertia subfield (maximal unramified extension) of $K_{q}$ in $K_{q}\left(\zeta_{n}\right)$.

Then $\left(\left[K_{q}\left(\zeta_{n}\right): I\right], p\right)=1$. Because $\operatorname{Gal}\left(I \mid K_{q}\right)$ is isomorphic to a factor group of the finite abelian group $\operatorname{Gal}\left(K_{q}\left(\zeta_{n}\right) \mid K_{q}\right)$, then $\operatorname{Gal}\left(I \mid K_{q}\right)$ is isomorphic to a subgroup of $\operatorname{Gal}\left(K_{q}\left(\zeta_{n}\right) \mid K_{q}\right)$ and in particular the $p$-Sylow subgroup of $\operatorname{Gal}\left(I \mid K_{q}\right)$ is isomorphic to the $p$-Sylow subgroup of $\operatorname{Gal}\left(K_{q}\left(\zeta_{n}\right) \mid K_{q}\right)$. But $I \mid K_{q}$ is an unramified extension and thus the $p$-Sylow subgroup of $\operatorname{Gal}\left(I \mid K_{q}\right)$ is cyclic. If $p \neq 2$, by the theorem of Goldschmidt and Isaacs [7], we have that $p \nmid m_{Q}(X)$, a contradiction, while if $p=2$ and $q$ is odd, we have that -1 is a sum of two squares in $K_{q}$ ([10, Lemma 2.2]) as $K_{q} \supset Q_{q}$. By Fein's theorem [5], again $2 \nmid m_{Q}(X)$. In either case we have a contradiction and have established the claim.

Thus $q_{i}$ is unramified in $K \mid Q$. By (*) we see that $E=F=Q$, and from Corollary 2. $e_{q_{1}}\left(K\left(\zeta_{q_{1}}\right) \mid K\right)_{p}=\left[K\left(\zeta_{q}\right): K\right]_{p}$. By Theorem 3 the result follows.

We observe that, it is sufficient to assume in Theorem 3 through Corollary 7 , that $K(X)$ is a nonreal field, rather than $m(X) \geqslant 3$, since that will guarantee that all infinite prime completions of the number field will be complex. Also the "splitting field", $L$, constructed in each case is a cyclic extension of $K$. 


\section{REFERENCES}

1. M. Benard and M. Schacher, The Schur subgroup. II, J. Algebra 22 (1972), 378-385.

2. G. Cornell, Abhyankar's lemma and the class group (Proc. Illinois Number Theory Conf.), Lecture Notes in Math., vol. 751, Springer-Verlag, Berlin and New York, 1979, pp. 82-88.

3. B. Fein, Minimal splitting fields for group representations, Pacific J. Math. 51 (1974), 427-431.

4. __ Minimal splitting fields for group representations. II, Pacific J. Math. 77 (1978), 445-449.

5. Schur indices and sums of squares, Proc. Amer. Math. Soc. 51 (1975), 31-34.

6. C. Ford, Groups which determine the Schur index of a representation, J. Algebra 57 (1979), 339-354.

7. D. Goldschmidt and I. Isaacs, Schur indices in finite groups, J. Algebra 33 (1975), 191-199.

8. H. Hasse, Number theory, Springer-Verlag, Berlin and New York, 1980.

9. R. Mollin, Splitting fields and group characters, J. Reine Angew. Math. 315 (1980), 107-114.

10. E. Spiegel and A. Trojan, On semi-simple group algebras. II, Pacific J. Math. 66 (1976), 553-559.

11. T. Yamada, The Schur subgroup of the Brauer group, Lecture Notes in Math., vol. 397, SpringerVerlag, Berlin and New York, 1974.

Department of Mathematics, University of Connecticut, StorRs, Connecticut 06268

Department of Mathematics, Atkinson College, York University, Downsview, Ontario M3J IP3, CANADA 